\title{
Extrahepatic Feeding of HCC Limits the Use of TACE? Evidences from Literature and Clinical Experience
}

\author{
Alessandra Tovoli ${ }^{1}$, Lucia Napoli ${ }^{1}$, Marianna Mastroroberto ${ }^{2}$, Matteo Renzulli ${ }^{2}$, Manuel Tufoni ${ }^{1}$, \\ Carmen Serena Ricci ${ }^{1}$, Rita Golfieri ${ }^{3}$, Giovanni Brandi ${ }^{4^{*}}$ \\ ${ }^{1}$ Department of Medical and Surgical Sciences, Alma Mater Studiorum University of Bologna, Bologna, Italy; ${ }^{2}$ Department of \\ Internal Medicine and Digestive Diseases, Azienda Ospedaliera-Universitaria Policlinico Sant' Orsola-Malpighi, Bologna, Italy; \\ ${ }^{3}$ Department of Internal Medicine and Gastroenterology, Azienda Ospedaliera-Universitaria Policlinico Sant' Orsola-Malpighi, \\ Bologna, Italy; ${ }^{4}$ Department of Experimental, Diagnostic and Speciality Medicine, Alma Mater Studiorum University of Bologna, \\ Bologna, Italy. \\ Email: "giovanni.brandi@unibo.it
}

Received December $21^{\text {st }}, 2012$; revised January $23^{\text {rd }}, 2013$; accepted February $1^{\text {st }}, 2013$

\begin{abstract}
Trans-arterial chemoembolization (TACE) has been established as the standard of care for patients with intermediate stage of multinodular hepatocellular carcinoma (HCC), according to Barcelona Clinic Liver Cancer (BCLC) classification, and no extra-hepatic diffusion. The efficacy and safety of the procedure are related to the possibility of performing a superselective catheterization of the branches of the hepatic artery responsible for the vascularisation of the tumour. However, in some cases, the vascularisation of the nodules can be complex and arterial supply can derive from vessels originating from the extra-hepatic circulation. This condition, called extra-hepatic feeding, is not a rare finding, and can hamper the therapeutic efficacy of TACE. When investigating a candidate for TACE, anamnestic and radiological elements suggestive for the presence of extra-collateral arteries should be known and taken into account. Once diagnosed, although extra-hepatic feeding does not represent an absolute contraindication to TACE, it requires, in each case, a careful benefit-risk assessment, being impossible to establish a standard procedure. We here present a review of the available literature and a paradigmatic case of multifocal $\mathrm{HCC}$ with an extrahepatic feeding.
\end{abstract}

Keywords: HCC; Extrahepatic Feeding; TACE

\section{Introduction}

Hepatocellular carcinoma (HCC) is the fifth malignancy in the world, with a steadily increasing incidence, the third cause of death for cancer, and accounts for about $7 \%$ of all malignancy in the world. HCC represents more than $90 \%$ of primary liver cancers [1]. Cirrhosis is the most important risk factor for $\mathrm{HCC}$ and about one-third of cirrhotic patients will develop HCC during their lifetime [2]. Although cirrhosis from hepatotopic viruses is more prone to HCC, all other etiologic forms of cirrhosis are complicated by tumor formation [3].

Liver parenchyma has a dual blood supply from both portal vein and hepatic artery, but the vascularisation of HCC nodules is derived solely from the arterial system as a result of the high neoangiogenic activity of this tumour. In the vast majority of the cases, HCC is vascularised by branches of the hepatic artery [4,5], although it may happen that some HCC nodules exhibit an abnormal vascularisation from extra-hepatic collateral arteries (ExCAs),

"Corresponding author. even if the hepatic artery is patent [6,7]. This condition, called extra-hepatic feeding, is not uncommon and it has a clinical relevance, mostly because it can compromise the efficacy of the treatment with trans-arterial chemioembolisation (TACE) [7].

TACE is the first line therapy for unresectable HCC in the intermediate stage (stage B of Barcelona Clinic Liver Cancer Classification), represented by multinodular HCC (exceeding the Milan criteria), without any vascular invasion or extrahepatic tumoral spread and preserved liver function $[3,8,9]$. TACE consists in the intra-arterial infusion of chemotherapeutic agents, usually mixed with $\mathrm{Li}$ piodol, followed by the embolization of the arterial afferent vessels of the tumoral lesion [9-11], resulting in a marked reduction of the arterial flow and necrosis of the tumoral cells $[12,13]$. Efficacy and safety of the procedure are related to the possibility of performing a superselective catheterization of the branches of the artery responsible for the vascularisation of the tumor [14].

However, efficacy and safety of TACE may be hampered by the presence of ExCA, since part of the tumour 
will be not reach by the chemioterapic and Lipiodol, remaining thus viable independently of treatment. Therefore, it appears important to consider the possible presence of ExCA in candidates to TACE that show anamnestic and radiological elements suggestive of it [15-19] and a selective angiography of all suspected collaterals should be done to confirm the diagnosis [20,21]. Once established, ExCA itself does not represent an absolute contraindication to TACE, but it requires, in each case, a careful benefit-risk assessment and considerable technical skill in performing the procedure.

We here present a review of the literature about ExCA and a paradigmatic case of a patient with a long history of recurrent HCC.

\section{Features Associated to Extra-Hepatic Feeding of HCC by ExCA}

Several clinical and pathological features have been associated to the extrahepatic feeding with ExCA (Table 1).

Exophytic and extracapsular tumors have been proposed to be more frequently associated with ExCA [20]. Furthermore, HCC located in the bare area of the liver or those in direct contact with suspensory ligaments as well as tumours which frankly invade abdominal organs, such as stomach, colon and kidney, appear to be linked to extrahepatic feeding $[6,20,22]$.

Beside location, the other major element associated with ExCA formation is the tumor size: tumours greater than $6 \mathrm{~cm}$ have a $63 \%$ of probability to have an ExCA,

Table 1. Features associated with the presence of extrahepatic feeding by extrahepatic collateral arteries (ExCAs). CT computerized tomography, TACE Trans-arterial chemoembolization.

\begin{tabular}{ll}
\hline & Elements suggesting ExCA \\
\hline Tumor & Exophytic \\
& Big size $(4-6 \mathrm{~cm})$ \\
& Extracapsular \\
& Bare area of the liver \\
Tumor location & Contact with suspensory ligaments \\
& Invasion of abdominal organs \\
Previous procedures & $\begin{array}{l}\text { Multiple TACE sessions } \\
\text { dissection of the hepatic arterial } \\
\text { branches }\end{array}$ \\
& $\begin{array}{l}\text { Hypertrophied extrahepatic } \\
\text { collateral vessel on a CT scan } \\
\text { Peripheral defect of iodized oil } \\
\text { retention within a tumor seen } \\
\text { during chemoembolization or on a } \\
\text { follow-up CT scan }\end{array}$ \\
\hline
\end{tabular}

and there is a general increase of the prevalence for tumour sized in the range of $4-6 \mathrm{~cm}[20,23]$. Li et al. showed that ExCAs were present in the $43 \%$ of their population of patients with unresectable HCC [24].

In about $5 \%$ of cases, the development of an ExCA occurs as a result of procedures that interrupt the blood supply to liver segments for prolonged periods of time, as in the case of surgical resections and dissection of the hepatic arterial branches secondary to endovascular manoeuvres [25]. Okazaki et al. demonstrated that one or more recurrent HCCs with ExCA were found in 38\% of their population of post-hepatectomy patients [26].

Also repeated TACEs for recurrent HCC are related with an increased probability of extrahepatic feeding due to ExCA development [20], which may decrease the efficacy of other TACE [27]. The incidence of ExCA onset increases with the number of repeated procedures: $17.9 \%$ after the fourth TACE and $56.4 \%$ after the fifth or the sixth chemoembolization (56.4\%) [27].

Surgical resection and TACE cause an obliteration of segmental hepatic arterial branches with a lack of arterial perfusion in the corresponding liver portion and stimulate a compensatory angiogenetic process, favouring revascularisation of the ischemic area $[22,28,29]$.

\section{Diagnosis of the ExCA Feeding HCC}

Due to the liver proximity to the diaphragm, the right inferior phrenic artery is the most frequent collateral vessel that supply HCC (in the $50 \%$ of the cases), especially when the mass is located in the S7 or S2-3 liver segments [30]. Tumours located in the ventral hepatic area, close to the anterior abdominal wall, can be supplied by the internal mammary artery, such as by the lower intercostal, subcostal and lumbar artery. Furthermore, when HCC grows until it directly invades intraabdominal organs, the arterial supply can derive from the gastric artery or branches of the superior mesenteric artery [30]. In the same way, adrenal arteries and renal capsular arteries can feed tumours that extend inferomedially [31], while HCC next to the gallbladder bed can be predisposed to a cystic artery supply [32]. Finally, previous laparotomic abdominal operations can be the cause of postoperative peritoneal adhesions, that consequently can cause an extrahepatic feeding in several portions of the liver, due to the omentum mobility [33]. Table 2 shows the list of the more frequent ExCAs responsible for HCC extrahepatic feeding.

Extrahepatic feeding of HCC should be early suspected during the diagnostic work-up. Large size, extracapsular or exophytic tumours, as well as an asymmetrical hypertrophy of specific collateral vessels, should always prompt the suspicion of ExCA. Moreover, the existence of ExCA should be suspected when the CT scan 
Table 2. Suspected origin of extrahepatic collateral arteries (ExCAs) on the basis of hepatocellular carcinoma (HCC) site.

\begin{tabular}{|c|c|}
\hline \multicolumn{2}{|c|}{ Suspected origin of ExCA on the basis of HCC site } \\
\hline HCC site & Suspected origin of ExCA \\
\hline Bare area of the liver & Right inferior phrenic artery and/or the right adrenal artery \\
\hline Superoanterior portion of the liver & Internal mammary artery \\
\hline Contact with the right kidney & Right renal artery and right adrenal artery \\
\hline Contact with omental fat & Gastroduodenal artery and of the right and left gastroepiploic arteries \\
\hline Invasion or contact with the right lateral thoracic wall & Lower intercostal arteries \\
\hline Left lateral segment of the liver & Gastric arteries \\
\hline Contact with the colon & Colic branch of the superior mesenteric artery \\
\hline
\end{tabular}

post-TACE shows a peripheral Lipiodol retention defect in the treated tumoral lesion [25] or in case of a persistent elevation of serum $\alpha$-fetoprotein level even after successful chemoembolization via the hepatic arteries and no evidence of new active nodules [5,32,34-36].

When ExCA is suspected, the diagnosis can be confirmed by an angio-CT or angio-MRI. However, a selective angiography of the suspected collaterals arteries before performing TACE is mandatory to confirm the diagnosis of ExCA [20,21].

\section{Treatment with TACE in Presence of an Extrahepatic Feeding by ExCA}

The presence of ExCA feeding HCC nodules may lead to a poor therapeutic response of TACE [21]. It appears obvious that in presence of ExCA a part of the tumour will remain viable as spared by the chemotherapic agent mixed with Lipiodol and by the ischemia caused by the occlusion of the afferent artery.

However, extra-hepatic feeding does not represent by itself a contraindication to TACE either when the regular hepatic vascularisation is partially or completely replaced. In the majority of the cases, hepatic artery and ExCA coexist and most patients with a collateral supply have a widely patent hepatic artery; while only about $5 \%$ of patients have proximal hepatic artery occlusion [25].

TACE can be performed, with some precautions, by performing the chemoembolization of the extrahepatic collateral vessels if technically feasible $[20,21]$. Two approaches can be followed based on the results of the angiographic study. If reflux of material towards extrahepatic organs exists, TACE should be performed by closing firstly the collateral vessels with a superselective embolization, using embolic material or spirals [14]. Doing that, the flow to extra-hepatic organs can be minimized and TACE can be subsequently performed through the hepatic artery, without extrahepatic implications. Otherwise, if there is no extrahepatic reflux, chemoembolization can be done directly through the extrahepatic vessel using co- axial microcatheters to prevent arterial spasm [21]. Unfortunately, in the case of very large HCC, the tumour can be vascularised by multiple collateral arterial vessels and treatment efficacy is quite difficult to be achieved.

However, TACE through the collateral vessels can favour the onset of complications of "non-target" organs. Skin problems such as itching, erythema, and necrosis, are related to embolization internal mammary, intercostal or lumbar artery [37,38], while ischemic cholecystitis is related to embolization of the cystic artery [36]. Gastrointestinal erosion and even ulceration can result by gastric, omental, and colic branch artery embolization [39]. Shoulder pain, pleural effusion, or basal atelectasis are related to chemoembolization of the inferior phrenic artery. Spinal cord injury up to paraplegia has been described with adventitious embolization of branches of spinal collateral vessels derived from the intercostal or lumbar artery [25]. Finally, embolism/pulmonary infarction and splenic infarction have been also reported $[37,40,41]$. Therefore, a careful assessment balancing probability of response and possibility of causing severe side-effects, should be individually considered when planning to perform TACE trough collates vessels, as the extreme variability of ExCA does not allow to establish a standard approach [21].

\section{A Paradigmatic Case of Extrahepatic Feeding}

A 53-year-old man, suffering from well-compensated HBV-related cirrhosis with a persistent suppression of viral replication by the nucleoside analogues therapy (adefovir $10 \mathrm{mg}$ /day, lamivudine $100 \mathrm{mg} /$ day), came to our attention in January 2012 for persistence of HCC. Liver function was always preserved (Child-Pugh A) and he underwent to several treatments for recurrent HCC.

In 2008 a wedge resection of a $38 \mathrm{~mm}$ HCC nodule at VII liver segment was performed. In 2011 a MRI showed 2 nodules of $\mathrm{HCC}$ at the VIII and at the V segments of 9 
$\mathrm{mm}$ and $7 \mathrm{~mm}$, respectively. Due to the impossibility of the visualisation at CEUS, and to the proximity of one lesion to the hepatic bare area, both nodules were treated with TACE. After one month, a follow-up CT scan demonstrated that the HCC lesions of the right hepatic lobe had been successfully treated and revealed a new lesion at the IV segment $(14 \mathrm{~mm})$. Thereafter, this last lesion was surgically resected on December 2011. He always refused the chance of a liver transplant.

An additional MRI, performed on January 2012, revealed three new HCC nodules of $34 \times 43 \mathrm{~mm}$ and $15 \times$ $16 \mathrm{~mm}$ at the VIII segment, and of $14 \times 13 \mathrm{~mm}$ at the VI segment. Due to the multinodular HCC and to the proximity of two of the lesions to the diaphragm, another TACE was planned. During the angiographic study, just the smaller lesion of the VIII was identified and then treated along with the one of the VI segment.

The patient came to our attention when a new CT scan revealed the persistence of the larger HCC lesion of the VIII segment, which had reached the size of $36 \times 44 \mathrm{~mm}$, in the absence of vascular invasion. The patient had a well preserved liver function (Child Pugh was 6 and MELD score was 8 ) and $\alpha$-fetoprotein was $32 \mathrm{ng} / \mathrm{ml}$.

Due to the proximity of the nodule to the diaphragm, percutaneous techniques were ruled out and it was decided to perform a further therapeutic attempt with TACE (the third in his history) and to add a selective study of potential collateral arteries, in order to rule out the presence of ExCA of the nodule. While the angiographic study of the hepatic artery did not show any hypervascular parenchymal area, the subsequent injection of contrast media in the right phrenic artery revealed the nodule of the VIII segment in the hepatic dome (Figure 1). There was no evidence of reflux of material towards extra-hepatic organs. Afterwards, the superselective catheterization of the right phrenic artery was performed and a cocktail with Farmarubicin and Lipiodol was infused, followed by embolization with spongel. The treated nodule was completely excluded by vascularisation, without complications.

The patient tolerated the procedure well with just a mild increase of aspartate aminotrasferase (70 U/lt) and alanine aminotrasferase levels (80 U/lt), and was discharged from the hospital three days after. One month after the procedure, a follow up MRI showed the absence of any tumoral activity and demonstrated the efficacy of the selective TACE procedure, his $\alpha$-fetoprotein was 13 $\mathrm{ng} / \mathrm{ml}$.

To date, the patient is still followed in our outpatient clinic without evidence of $\mathrm{HCC}$ recurrence.

\section{Conclusions}

The case above described is paradigmatic of the clinical situation of extrahepatic feeding. Although the HCC no-

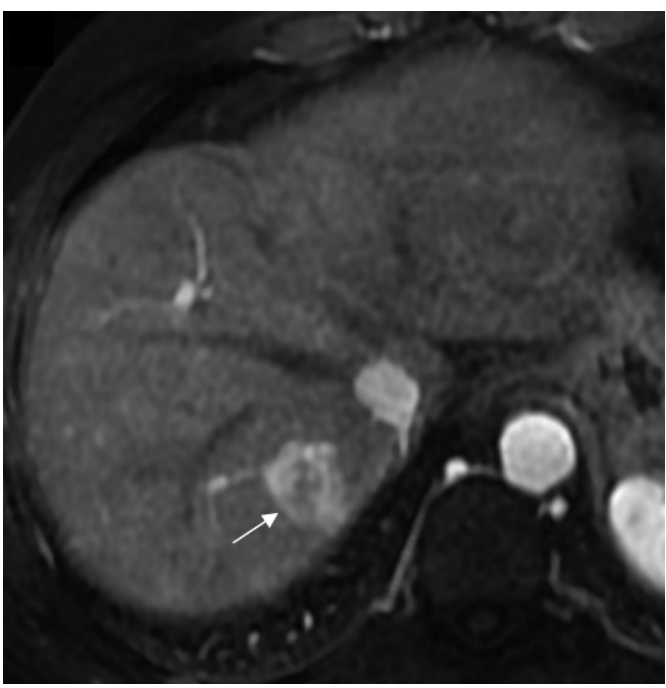

(a)

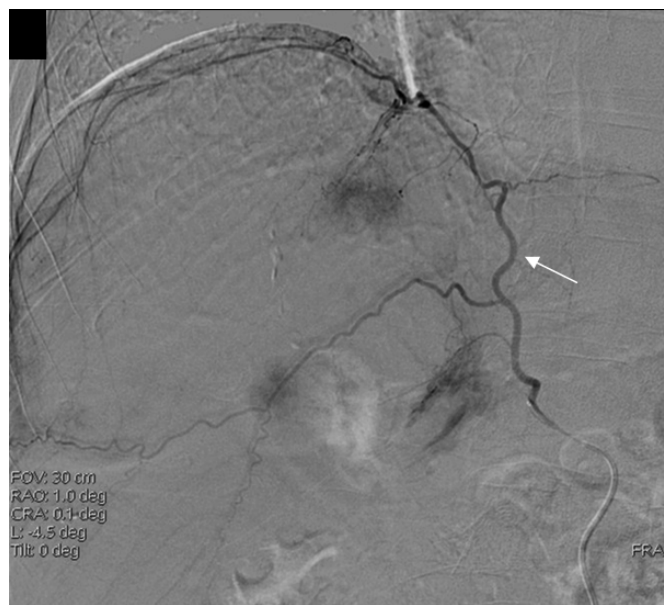

(b)

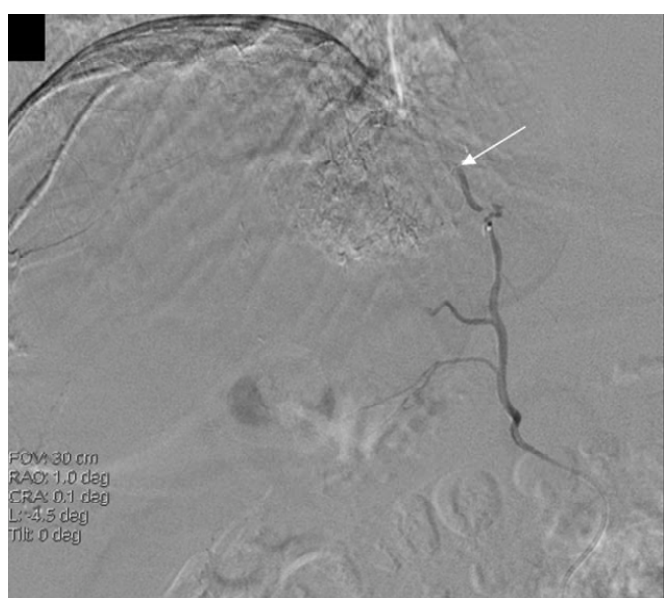

(c)

Figure 1. MRI image showing HCC before TACE treatment (a) and angiography showing the extrahepatic feeding by ExCA originating from the phrenic artery (b), which was successfully chemioembolized (c). HCC, hepatocellular carcinoma; TACE, Trans-arterial chemoembolization; ExCA, extrahepatic collateral artery. 
dule was well visualised by a CT scan, it was not detectable on the angiographic study during the second TACE, and the treatment failed with persistency of high $\alpha$-fetoprotein serum level. This particular behaviour is highly suggestive of ExCA and should have been noticed; furthermore, there were some other elements that should have raised the suspicion of the formation of collateral extrahepatic vessels [21]. Indeed, the nodule was situated in the hepatic dome, close to the bare area of the liver and it had relatively high dimensions. Moreover, the patient underwent to a previous liver resection and TACEs on the same area, that could have caused damages to the vascular system of branches of the hepatic artery. All these elements are highly suggestive for the development of ExCA [40].

Basing on these considerations, a selective angio-graphic study of the collateral vessels, particularly of the right inferior phrenic artery, considering the HCC location close to the diaphragm, was warranted and allowed to perform a successful TACE leading eventually to the complete necrosis of the nodule $[21,40]$.

In conclusion, ExCA is not a rare finding, particularly in patients already exposed to repeated TACE treatments and when the HCC is large or peripherally located. The awareness of ExCA is of crucial importance because its diagnosis represents the pre-requisite for TACE success [7,20,21,42]. Once established, ExCA itself does not represent an absolute contraindication to TACE, but it requires a careful benefit-risk assessment in each case and considerable technical skill in performing the procedure.

\section{REFERENCES}

[1] J. M. Llovet, "Updated Treatment Approach to Hepatocellular Carcinoma," Journal of Gastroenterology, Vol. 40, No. 3, 2005, pp. 225-235. doi:10.1007/s00535-005-1566-3

[2] A. Sangiovanni, G. M. Prati, P. Fasani, et al., "The Natural History of Compensated Cirrhosis Due to Hepatitis C Virus: A 17-Year Cohort Study of 214 Patients," Hepatology, Vol. 43, No. 6, 2006, pp. 1303-1310. doi:10.1002/hep. 21176

[3] European Association for Study of Liver, European Organization for Research and Treatment of Cancer, "EASLEORTC Clinical Practice Guidelines: Management of Hepatocellular Carcinoma," European Journal of Cancer, Vol. 48, No. 5, 2012, pp. 599-641. doi:10.1016/j.ejca.2011.12.021

[4] A. Guglielmi, A. Ruzzenente, S. Pachera, et al., "Comparison of Seven Staging Systems in Cirrhotic Patients with Hepatocellular Carcinoma in a Cohort of Patients Who Underwent Radiofrequency Ablation with Complete Response," American Journal of Gastroenterology, Vol. 103, No. 3, 2008, pp. 597-604. doi:10.1111/j.1572-0241.2007.01604.x

[5] J. H. Kim, J. W. Chung, J. K. Han, et al., "Transcatheter
Arterial Embolization of the Internal Mammary Artery in Hepatocellular Carcinoma," Journal of Vascular and Interventional Radiology, Vol. 6, No. 1, 1995, pp. 71-77. doi:10.1016/S1051-0443(95)71059-4

[6] L. F. Cheng, K. F. Ma, W. C. Fan, et al., "Hepatocellular Carcinoma with Extrahepatic Collateral Arterial Supply," Journal of Medical Imaging and Radiation Oncology, Vol. 54, No. 1, 2010, pp. 26-34. doi:10.1111/j.1754-9485.2010.02134.x

[7] S. B. Paul, S. R. Gamanagatti, A. Mukund, et al., "Transarterial Chemoembolization for Hepatocellular Carcinoma: Significance of Extrahepatic Collateral Supply," Indian Journal of Cancer, Vol. 48, No. 3, 2011, pp. 339-344. doi:10.4103/0019-509X.84941

[8] C. M. Lo, H. Ngan, W. K. Tso, et al., "Randomized Controlled Trial of Transarterial Lipiodol Chemoembolization for Unresectable Hepatocellular Carcinoma," Hepatology, Vol. 35, No. 5, 2002, pp. 1164-1171. doi:10.1053/jhep.2002.33156

[9] J. M. Llovet, M. I. Real, X. Montaña, et al., "Arterial Embolisation or Chemoembolisation versus Symptomatic Treatment in Patients with Unresectable Hepatocellular Carcinoma: A Randomised Controlled Trial," Lancet, Vol. 359, No. 9319, 2002, pp. 1734-1739. doi:10.1016/S0140-6736(02)08649-X

[10] R. Lencioni, "Chemoembolization for Hepatocellular Carcinoma," Seminars in Oncology, Vol. 39, No. 4, 2012, pp. 503-509. doi:10.1053/j.seminoncol.2012.05.004

[11] T. Gunji, N. Kawauchi, M. Akahane, et al., "Long-Term Outcomes of Transcatheter Arterial Chemoembolization with Autologous Blood Clot for Unresectable Hepatocellular Carcinoma," International Journal of Oncology, Vol. 21, No. 2, 2002, pp. 427-432.

[12] J. A. Marrero, R. J. Fontana, A. Barrat, et al., "Prognosis of Hepatocellular Carcinoma: Comparison of 7 Staging Systems in an American Cohort," Hepatology, Vol. 41, No. 4, 2005, pp. 707-716. doi:10.1002/hep.20636

[13] T. Konno, "Targeting Cancer Chemotherapeutic Agents by Use of Lipiodol Contrast Medium," Cancer, Vol. 66, No. 9, 1990, pp. 1897-1903.

doi:10.1002/1097-0142(19901101)66:9<1897::AID-CNC R2820660907>3.0.CO;2-J

[14] R. Golfieri, A. Cappelli, A. Cucchetti, et al., "Efficacy of Selective Transarterial Chemoembolization in Inducing Tumor Necrosis in Small $(<5 \mathrm{~cm})$ Hepatocellular Carcinomas," Hepatology, Vol. 53, No. 5, 2011, pp. 15801589. doi:10.1002/hep. 24246

[15] D. Y. Lin, Y. F. Liaw, T. Y. Lee, et al., "Hepatic Arterial Embolization in Patients with Unresectable Hepatocellular Carcinoma-A Randomized Controlled Trial," Gastroenterology, Vol. 94, No. 2, 1988, pp. 453-456.

[16] G. Pelletier, A. Roche, O. Ink, et al., "A Randomized Trial of Hepatic Arterial Chemoembolization in Patients with Unresectable Hepatocellular Carcinoma," Journal of Hepatology, Vol. 11, No. 2, 1990, pp. 181-184. doi:10.1016/0168-8278(90)90110-D

[17] Groupe d'Etude et de Traitement du Carcinome Hépatocellulaire, "A Comparison of Lipiodol Chemoemboliza- 
tion and Conservative Treatment for Unresectable Hepatocellular Carcinoma," New England Journal of Medicine, Vol. 332, No. 19, 1995, pp. 1256-1261. doi:10.1056/NEJM199505113321903

[18] J. Bruix, J. M. Llovet, A. Castells, et al., "Transarterial Embolization versus Symptomatic Treatment in Patients with Advanced Hepatocellular Carcinoma: Results of a Randomized, Controlled Trial in a Single Institution," $\mathrm{He}$ patology, Vol. 27, No. 1, 1998, pp. 1578-1583. doi: 10.1002/hep. 510270617

[19] G. Pelletier, M. Ducreux, F. Gay, et al., "Treatment of Unresectable Hepatocellular Carcinoma with Lipiodol Chemoembolization: A Multicenter Randomized Trial. Groupe CHC," Journal of Hepatology, Vol. 29, No. 1, 1998, pp. 129-134. doi:10.1016/S0168-8278(98)80187-6

[20] J. W. Chung, H. C. Kim, J. H. Yoon, et al., "Transcatheter Arterial Chemoembolization of Hepatocellular Carcinoma: Prevalence and Causative Factors of Extrahepatic Collateral Arteries in 479 Patients," Korean Journal of Radiology, Vol. 7, No. 4, 2006, pp. 257-266. doi:10.3348/kjr.2006.7.4.257

[21] H. C. Kim, J. W. Chung, W. Lee, et al., "Recognizing Extrahepatic Collateral Vessels that Supply Hepatocellular Carcinoma to Avoid Complications of Transcatheter Arterial Chemoembolization," Radiographics, Vol. 25, 2005, pp. S25-S39. doi:10.1148/rg.25si055508

[22] C. R. Davis, "Interventional Radiological Treatment of Hepatocellular Carcinoma," Cancer Control, Vol. 17, No. 2, 2010, pp. 87-99.

[23] S. Miyayama, M. Yamashiro, M. Okuda, et al., "The March of Extrahepatic Collaterals: Analysis of Blood Supply to Hepatocellular Carcinoma Located in the Bare Area of the Liver After Chemoembolization," CardioVascular and Interventional Radiology, Vol. 33, No. 3, 2010, pp. 513-522. doi:10.1007/s00270-009-9697-1

[24] C. Li, Y. Guo, G. Tian, et al., "Extrahepatic Arterial Blood Supply of Hepatocellular Carcinoma and Interventional Treatment," Chinese Journal of Oncology, Vol. 24, No. 2, 2002, pp. 163-166.

[25] J. W. Chung, J. H. Park, J. K. Han, et al., "Transcatheter Oily Chemoembolization of the Inferior Phrenic Artery in Hepatocellular Carcinoma: The Safety and Potential Therapeutic Role," Journal of Vascular and Interventional Radiology, Vol. 9, No. 3, 1998, pp. 495-500. doi:10.1016/S1051-0443(98)70306-9

[26] M. Okazaki, S. Yamasaki, H. Ono, et al., "Chemoembolotherapy for Recurrent Hepatocellular Carcinoma in the Residual Liver after Hepatectomy," Hepatogastroenterology, Vol. 40, No. 4, 1993, pp. 320-323.

[27] Y. L. Wang, M. H. Li, Y. S. Cheng, et al., "Influential Factors and Formation of Extrahepatic Collateral Artery in Unresectable Hepatocellular Carcinoma," World Journal of Gastroenterology, Vol. 11, No. 17, 2005, pp. 26372642.

[28] K. H. Lee, K. B. Sung, D. Y. Lee, et al., "Transcatheter Arterial Chemoembolization for Hepatocellular Carcinoma: Anatomic and Hemodynamic Considerations in the Hepatic Artery and Portal Vein," Radiographics, Vol. 22, No. 5, 2002, pp. 1077-1091.
[29] C. Charnsangavej, V. P. Chuang, S. Wallace, et al., "Angiographic Classification of Hepatic Arterial Collaterals," Radiology, Vol. 144, No. 3, 1982, pp. 485-494.

[30] Y. Okino, H. Kiyosue, S. Matsumoto, et al., "Hepatocellular Carcinoma: Prediction of Blood Supply from Right Inferior Phrenic Artery by Multiphasic CT," Journal of Computer Assisted Tomography, Vol. 27, No. 3, 2003, pp. 341-346. doi:10.1097/00004728-200305000-00007

[31] T. Shibata, N. Kojima, T. Tabuchi, et al., "Transcatheter Arterial Chemoembolization through Collateral Arteries for Hepatocellular Carcinoma after Arterial occlusion," Radiation Medicine, Vol. 16, No. 4, 1998, pp. 251-256.

[32] S. Miyayama, O. Matsui, H. Nishida, et al., "Transcatheter Arterial Chemoembolization for Unresectable Hepatocellular Carcinoma Fed by the Cystic Artery," Journal of Vascular and Interventional Radiology, Vol. 14, No. 9, 2003, pp. 1155-1161. doi:10.1097/01.RVI.0000086534.86489.10

[33] J. Y. Won, D. Y. Lee, J. T. Lee, et al., "Supplemental Transcatheter Arterial Chemoembolization through a Collateral Omental Artery: Treatment for Hepatocellular Carcinoma," CardioVascular and Interventional Radiology, Vol. 26, No. 2, 2003, pp. 136-140. doi:10.1007/s00270-002-2629-y

[34] M. Nakai, M. Sato, N. Kawai, et al., "Hepatocellular Carcinoma: Involvement of the Internal Mammary Artery," Radiology, Vol. 219, No. 1, 2001, pp. 147-152.

[35] S. I. Park, D. Y. Lee, J. Y. Won, et al., "Extrahepatic Collateral Supply of Hepatocellular Carcinoma by the Intercostal Arteries," Journal of Vascular and Interventional Radiology, Vol. 14, No. 4, 2003, pp. 461-468. doi:10.1097/01.RVI.0000064856.87207.1E

[36] J. W. Chung, J. H. Park, J. K. Han, et al., "Hepatic Tumors: Predisposing Factors for Complications of Transcatheter Oily Chemoembolization," Radiology, Vol. 198, No. 1, 1996, pp. 33-40.

[37] R. Arora, M. C. Soulen and Z. J. Haskal, "Cutaneous Complications of Hepatic Chemoembolization via Extrahepatic Collaterals," Journal of Vascular and Interventional Radiology, Vol. 10, No. 10, 1999, pp. 1351-1356. doi:10.1016/S1051-0443(99)70242-3

[38] J. H. Lee, C. Y. Chon, S. H. Ahn, et al., "An Ischemic Skin Lesion after Chemoembolization of the Right Internal Mammary Artery in a Patient with Hepatocellular Carcinoma," Yonsei Medical Journal, Vol. 42, No. 1, 2001, pp. 137-141.

[39] M. Hirakawa, M. Iida, K. Aoyagi, et al., "Gastroduodenal Lesions after Transcatheter Arterial Chemo-Embolization in Patients with Hepatocellular Carcinoma," American Journal of Gastroenterology, Vol. 83, No. 8, 1988, pp. 837-840.

[40] S. Miyayama, O. Matsui, K. Taki, et al., "Extrahepatic Blood Supply to Hepatocellular Carcinoma: Angiographic Demonstration and Transcatheter Arterial Chemoembolization," CardioVascular and Interventional Radiology, Vol. 29, No. 1, 2006, pp. 39-48. doi:10.1007/s00270-004-0287-y

[41] I. Sakamoto, N. Aso, K. Nagaoki, et al., "Complications 
Associated with Transcatheter Arterial Embolization for Hepatic Tumors," Radiographics, Vol. 18, No. 3, 1998, pp. 605-619.

[42] O. Matsui, M. Kadoya, J. Yoshikawa, et al., "Small Hepa- tocellular Carcinoma: Treatment with Subsegmental Transcatheter Arterial Embolization," Radiology, Vol. 188, No. 1, 1993, pp. 79-83. 